\title{
Moisture Effects on the Dielectric Properties of Soils
}

\author{
John O. Curtis
}

\begin{abstract}
Laboratory-measured dielectric property data and related electromagnetic wave propagation parameters are reported for a broad range of soil textures. The dielectric permittivity and phase velocity are shown to be very strong functions of volumetric soil moisture. A polynomial model for predicting soil moisture from permittivity measurements at $100 \mathrm{MHz}$ is presented.
\end{abstract}

Index Terms-Conductivity, dielectric properties, permitivity, soil moisture.

\section{BACKGROUND}

$\mathbf{S}$ UCCESSFUL application of electromagnetic sensor systems to geophysical problems such as the measurement of soil moisture, analysis of terrain radar backscatter, location of subsurface anomalies, etc., require an appreciation of the attenuation of electromagnetic waves and the speed of those waves as they travel through the medium. Wave attenuation and speed are, in turn, controlled by the dielectric permittivity and electrical conductivity, or equivalently, the complex dielectric constant of the soil. Finally, these dielectric properties are going to be functions of signal frequency, the amount and nature of water in the soil, the chemistry of the soil, and the physical properties of the soil such as its structure and its mass density. This communication focuses on the question of how soil dielectric properties are affected by moisture.

Numerous researchers have collected soil electrical property data to support their particular programs of study. However, there does not seem to be a comprehensive data base of information on a wide range of soil textures that is readily available to the science and engineering community. Publications can be found in the literature that identify soil properties for a particular range of frequencies or for a limited number of soil textures [6], [11],[23], [24], [26], [28], [30]. Some of the more significant measurement programs have originated at the U.S. Geological Survey, Denver, CO, [18]-[21], the U.S. Army Cold Regions Research and Engineering Laboratory, Hanover, NH [10], [12], and the U.S. Army Waterways Experiment Station, Vicksburg, MS. [7], [8], [16], [17].

The author began in about 1989 to develop a measurement capability at the U.S. Army Engineer Research and Development Center (ERDC), formerly known as the Waterways Experiment Station, to help establish a data base of soil dielectric properties.

Manuscript received March 24, 1999; revised September 23, 1999. This work was supported by the Directorate of Research and Development, Headquarters of the U.S. Army Corps of Engineers, Vicksburg, MS, and was part of a more comprehensive examination of factors affecting the detectability of buried objects.

The author is with the U.S. Army Engineer Research and Development Center, Vicksburg, MS 39180-6199 USA (e-mail: curtisj@wes.army.mil).

Publisher Item Identifier S 0196-2892(01)00344-8.
The result of that effort was a coaxial transmission/reflection apparatus controlled by a Hewlett-Packard 8510C Vector Network Analyzer system [9]. The signal source spans a range of frequencies from $45 \mathrm{MHz}$ to $26.5 \mathrm{GHz}$. Soil samples were hand-packed into square cross section coaxial holders having lengths as short as $1.5 \mathrm{~cm}$ and as long as $10 \mathrm{~cm}$. Using this apparatus, over 700 different strings of data (each being a sweep of frequencies on one sample at one moisture condition) have been collected at a temperature of $20^{\circ} \mathrm{C}$. Data at other temperatures have also been collected but are not reported here.

\section{Mathematical Relationships}

Ampere's law dictates that the total current density in a medium is the sum of a conduction current in phase with the electric field and a displacement current in phase quadrature [15]. In a nonmagnetic material, the conduction current is controlled by the electrical conductivity of the medium and the strength of the electric field, while the displacement current is controlled by the dielectric permittivity and the time rate of change of the electric field. A similar interpretation could be made if the conductivity and dielectric permittivity were replaced by a complex relative dielectric permittivity

$$
\varepsilon=\varepsilon^{\prime}+i \varepsilon^{\prime \prime} .
$$

Conductivity, expressed in siemens per meter, is then replaced in the solution to the wave equation by the product of radian frequency and the imaginary part of the relative complex permittivity

$$
\sigma=\omega \varepsilon^{\prime \prime} \varepsilon_{0}
$$

where $\varepsilon_{0}$ is the permittivity of free space $\left(8.85 \times 10^{-12} \mathrm{~F} / \mathrm{m}\right)$.

For plain, exponentially-damped, harmonic, transverse wave propagation through a medium described by a complex permittivity, power attenuation, expressed in decibels per meter, can be written

power loss $=8.6859\left(\frac{\omega}{c}\right)\left[\frac{\varepsilon^{\prime}}{2}\left\{\sqrt{1+\left(\frac{\varepsilon^{\prime \prime}}{\varepsilon^{\prime}}\right)^{2}}-1\right\}\right]^{(1 / 2)}$

where $c$ is the speed of light in a vacuum.

Furthermore, plane waves of constant phase will propagate through the medium with a normalized velocity calculated by:

$$
\begin{aligned}
& \text { normalized phase velocity } \\
& =\frac{v}{c} \\
& =\left[\frac{\varepsilon^{\prime}}{2}\left\{\sqrt{1+\left(\frac{\varepsilon^{\prime \prime}}{\varepsilon^{\prime}}\right)^{2}}+1\right\}\right]^{-(1 / 2)}
\end{aligned}
$$




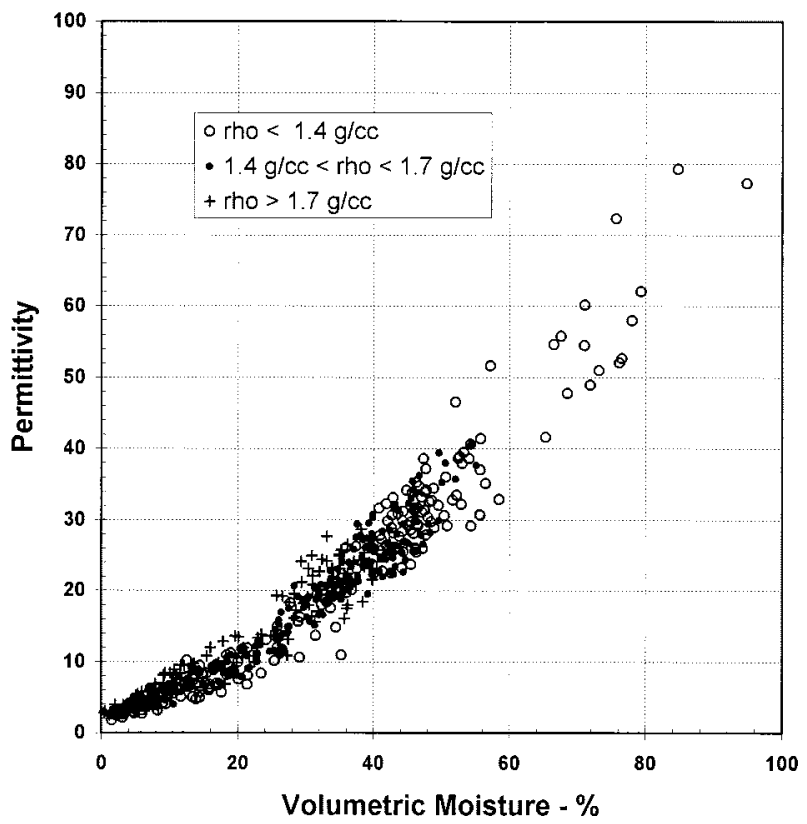

(a)

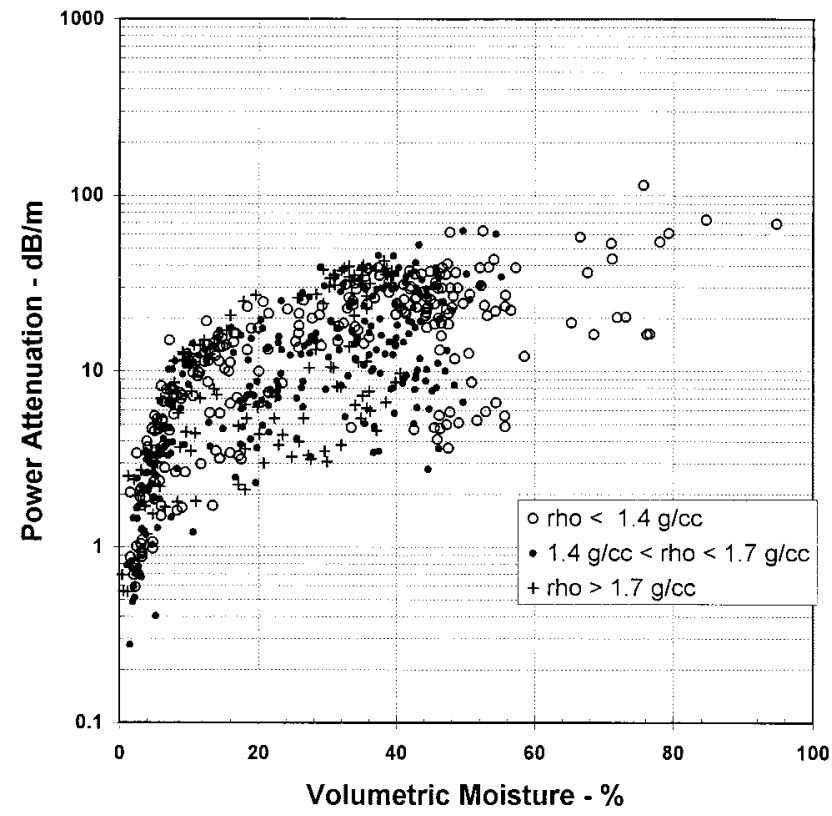

(c)

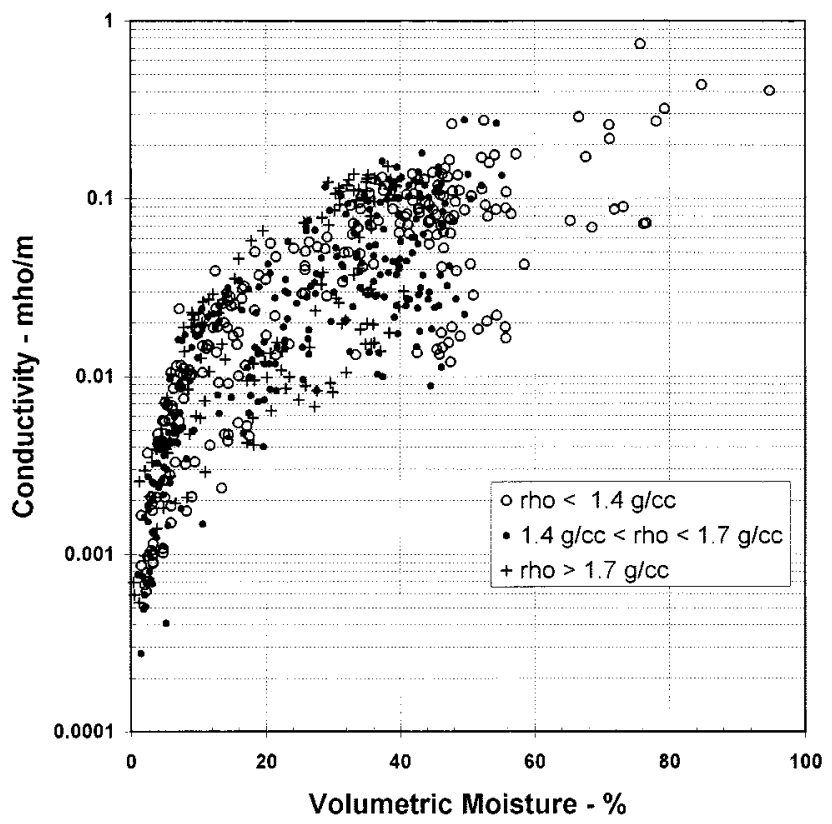

(b)

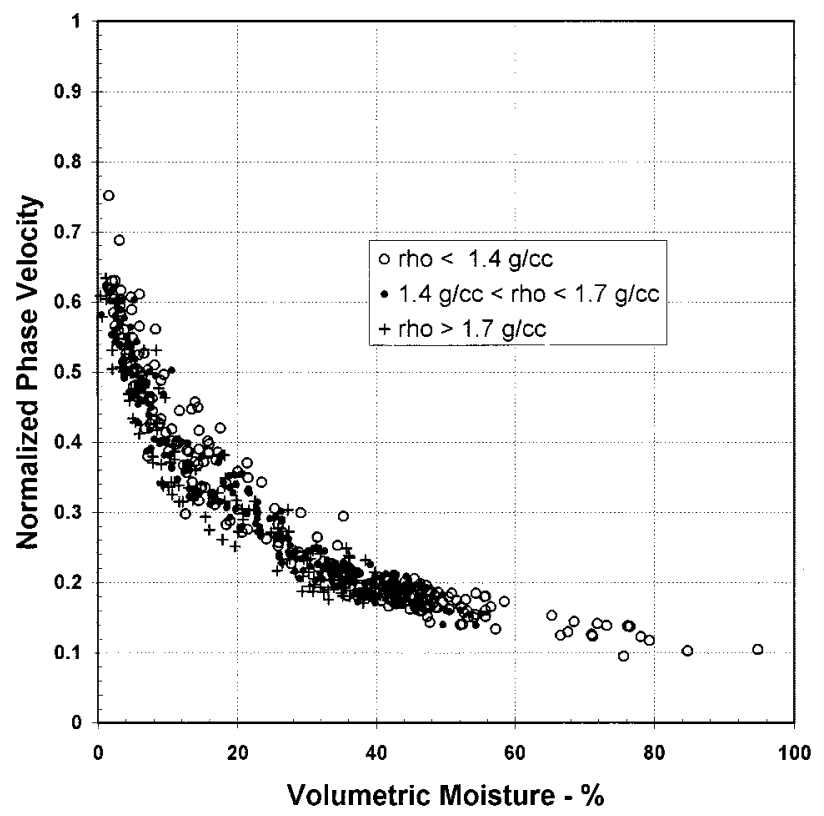

(d)

Fig. 1. Soil dielectric properties plotted against volumetric moisture at $100 \mathrm{MHz}$ and $20^{\circ} \mathrm{C}$.

\section{Electrical Properties OF SOILS AT $100 \mathrm{MHz}$}

Fig. 1 contains plots of the dielectric properties measured at ERDC versus volumetric moisture content at a frequency of 100 $\mathrm{MHz}$ and a sample temperature of $20^{\circ} \mathrm{C}$. The data have been sorted into one of three arbitrary sample dry density bands to allow observations on sample density effects to be made later in this section.

First of all, note that the real part of the complex dielectric constant (hereafter simply called the permittivity) and the normalized phase velocity are both very strong functions of volumetric moisture. One should not be surprised that the permittivity exhibits this behavior. After all, permittivity is a measure of the polarizability of the medium subjected to a time-varying electric field, and the fraction of the soil sample that possesses this polarizability property is that containing the water molecule dipoles. As for phase velocity, the plus sign in (4) has the effect of causing phase velocity to be more sensitive to permittivity than to the ratio, $\varepsilon^{\prime \prime} / \varepsilon^{\prime}$. For example, if one considers that $\varepsilon^{\prime \prime} / \varepsilon^{\prime}$ values lie generally between 0.0 and 1.0 , then the normalized phase velocity can vary between $1 / \sqrt{ } \varepsilon^{\prime}$ and $0.91 / \sqrt{ } \varepsilon^{\prime}$, in other words, sensitive mostly to permittivity.

While there is a strong relationship between permittivity and phase velocity and moisture content, there is no simple relationship between moisture content and conductivity and power attenuation. Obviously there are other factors involved in the 


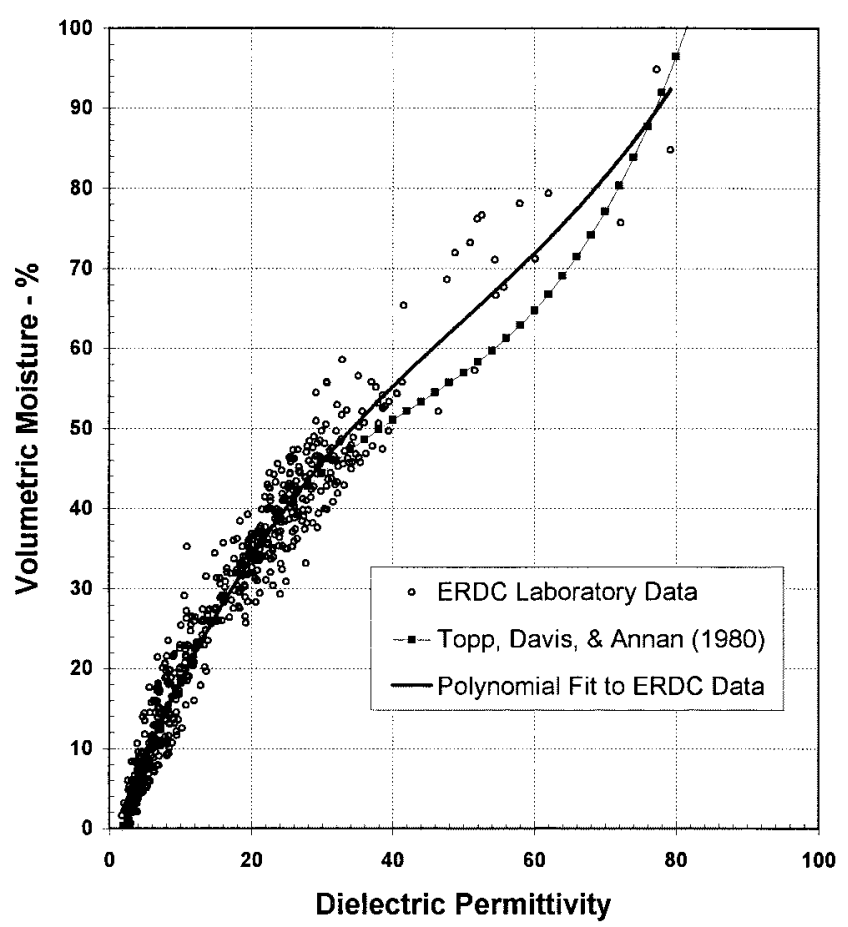

Fig. 2. Volumetric moisture-permittivity relationships at $100 \mathrm{MHz}$.

loss parameters besides just the amount of water in the sample. They must include such elements as the availability of salts in the soil matrix, the degree to which charged particles are bound to the soil matrix, the actual mechanisms of charge movement within the soil sample that might, in turn, include such factors as pore size distribution [1] and specific surface [29]. Development of a predictive model for soil conductivity will require a large amount of data on a variety of soil samples that have been thoroughly characterized from a physical and chemical perspective.

Fig. 1 also reveals that there appears to be no definitive relationship between the dielectric properties and sample dry density. This observation is not as apparent for permittivity and phase velocity as it is for conductivity and power attenuation. Intuitively, one would guess that increasing sample dry density (or decreasing the pore volume fraction) for a particular soil at a fixed moisture content would cause the dielectric properties of the soil to change smoothly in one direction or the other. However, with all soil textures presented on one chart, a clear relationship between dielectric properties and dry sample density is difficult to see. This is an area that begs for further investigation, although, when compared to the impact of moisture content, it is undoubtedly a second order effect.

\section{Using Dielectric Properties to Predict Volumetric SOIL MOISTURE CONTENT}

Given that there is such a strong dependence of dielectric permittivity on the volumetric moisture of a sample at any one frequency, it appears reasonable that one might be able to turn that concept around and use it to predict soil moisture from dielectric permittivity measurements. This is not an original concept. Several of the references listed at the end of this paper contain such models or describe soil moisture probes that have been

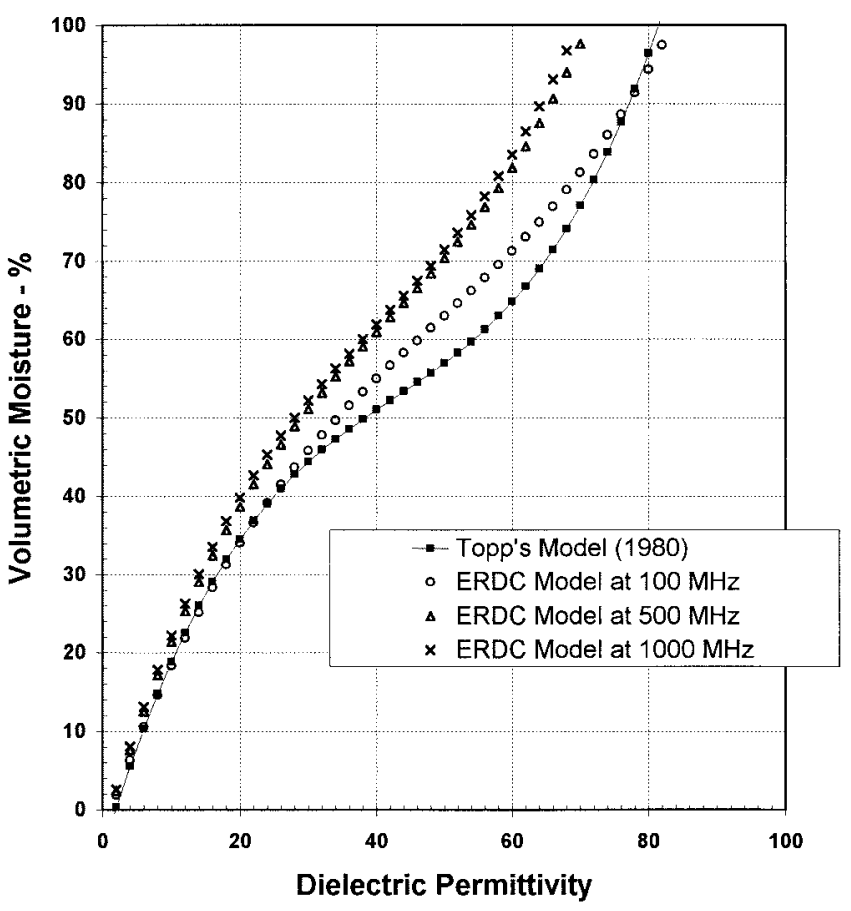

Fig. 3. Volumetric moisture-permittivity relationships at various frequencies.

developed on this principle [2]-[5], [7], [14], [22], [28]. The most widely used model is that of Topp, Davis, and Annan [28], which can be written

$$
\begin{aligned}
& \text { volumetric moisture }(\%) \\
& \quad=0.00043\left(\varepsilon^{\prime}\right)^{3}-0.055\left(\varepsilon^{\prime}\right)^{2}+2.92\left(\varepsilon^{\prime}\right)-5.3 .
\end{aligned}
$$

Fig. 2 is a chart of volumetric moisture values plotted against the dielectric permittivity measurements made at ERDC at 100 $\mathrm{MHz}$ and $20^{\circ} \mathrm{C}$. The thick solid line represents the least squares fit of a third order polynomial to the ERDC data

$$
\begin{aligned}
& \text { volumetric moisture(\%) } \\
& \quad=0.000237\left(\varepsilon^{\prime}\right)^{3}-0.03421\left(\varepsilon^{\prime}\right)^{2}+2.435\left(\varepsilon^{\prime}\right)-2.86 .
\end{aligned}
$$

The light line passing through squares was generated by the Topp, Davis, and Annan model, and below 25\% moisture content is virtually indistinguishable from the ERDC model.

Because in situ volumetric moisture contents rarely exceed $40 \%$, the conclusion of this study is that the Topp, Davis, and Annan model is a very good one for predicting volumetric moisture values in soil from dielectric permittivity measurements at a frequency of about $100 \mathrm{MHz}$. However, the Topp model is frequency independent, its fit being made to time domain reflectometry data. How then does frequency affect the soil moisture model?

Fig. 3 is a plot of model fits to the ERDC data base at three frequencies, namely, $100 \mathrm{MHz}, 500 \mathrm{MHz}$, and $1000 \mathrm{MHz}$. The Topp model is also reproduced on this figure. Clearly, the latter model is quite good at predicting soil moisture values at low frequencies and within a normal range of soil moistures. However, at higher frequencies, it can underpredict moisture values by as much as $10 \%$ or more. 


\section{REFERENCES}

[1] L. M. Arya and J. F. Paris, "A physicoempirical model to predict the soil moisture characteristic from particle-size distribution and bulk density data," Soil Sci. Soc. Amer. J., vol. 45, pp. 1023-1030, 1981.

[2] M. Baker and R. R. Allmaras, "System for automating and multiplexing soil moisture measurement by time-domain reflectometry," Soil Sci. Soc. Amer. J., vol. 54, pp. 1-6, Jan.-Feb. 1990.

[3] R. Birchak, C. G. Gardner, J. E. Hipp, and J. M. Victor, "High dielectric constant microwave probes for sensing soil moisture," Proc. IEEE, vol. 62, pp. 93-98, Jan. 1974

[4] D. R. Brunfeldt, "Theory and design of a field-portable dielectric measurement system," in Proc. IGARSS '87 Symp., Ann Arbor, MI, May 1987, pp. 559-563.

[5] R. Caldecott, M. Poirier, and D. E. Svoboda, "A radio frequency probe to measure soil electrical properties," The Ohio State Univ., Columbus, Final Rep. 715 616-4, Jan. 1985.

[6] J. E. Campbell, "Dielectric properties and influence of conductivity in soils at one to fifty megahertz," Soil Sci. Soc. Amer. J., vol. 54, pp. 332-341, Mar.-Apr. 1990.

[7] - Campbell Dielectric Probe Manual, V 1.03. Horseshoe Bend, IN: Campbell Consulting, Dec. 1998

[8] J. O. Curtis, C. A. Weiss Jr., and J. B. Everett, "Effect of soil composition on complex dielectric properties," U.S. Army Corps Eng. Waterways Experiment Station, Vicksburg, MS, Tech. Rep. EL-95-34, Dec. 1995.

[9] J. Curtis and R. Narayanan, "Effects of laboratory procedures on soil electrical property measurements," IEEE Trans. Instrum. Meas., vol. 47, pp. 1474-1480, Dec. 1998.

[10] J. O. Curtis, "A durable laboratory apparatus for the measurement of soil dielectric properties," IEEE Trans. Instrum. Meas., to be published.

[11] A. J. Delaney and S. A. Arcone, "Laboratory measurements of soil electric properties between 0.1 and $5 \mathrm{GHz}$," U.S. Army Corps Eng. Cold Regions Res. Eng. Lab., Hanover, NH, Rep. 82-10, Apr. 1982.

[12] M. T. Hallikainen, F. T. Ulaby, M. C. Dobson, M. A. El-Rayes, and L.-K. Wu, "Microwave dielectric behavior of wet soil-Part I: Empirical models and experimental observations," IEEE Trans. Geosci. Remote Sensing, vol. GE-23, pp. 25-34, Jan. 1985.

[13] P. Hoekstra and A. Delaney, "Dielectric properties of soils at UHF and microwave frequencies," J. Geophys. Res., vol. 79, pp. 1699-1708, Apr. 1974.

[14] J. E. Hipp, "Soil electromagnetic parameters as functions of frequency, soil density, and soil moisture," Proc. IEEE, vol. 62, pp. 98-103, Jan. 1974.

[15] T. J. Jackson, "Laboratory evaluation of a field-portable dielectric/soilmoisture probe," IEEE Trans. Geosci. Remote Sensing, vol. 28, no. 2, pp. 241-245, Mar. 1990

[16] J. D. Kraus, Electromagnetics, 3rd ed. New York: McGraw-Hill, 1984.

[17] J. R. Lundien, "Terrain analysis by electromagnetic means-Report 2: Radar responses to laboratory prepared soil samples," U.S. Army Corps Eng.Waterways Experiment Station, Vicksburg, MS, Tech. Rep. 3-693, Sept. 1966.
[18] _ - "Terrain analysis by electromagnetic means—Report 5: Laboratory measurement of electromagnetic propagation constants in the 1.0 to $1.5 \mathrm{GHz}$ microwave spectral region,” U.S. Army Corps Eng. Waterways Experiment Station, Vicksburg, MS, Tech. Rep. 3-693, Feb. 1971.

[19] G. R. Olhoeft, "Electrical properties of natural clay permafrost," Can. J. Earth Sci., vol. 14, no. 1, pp. 16-24, 1977

[20] — - "Low-Frequency electrical properties," Geophysics, vol. 50, no. 12, pp. 2492-2503, Dec. 1985.

[21] — "Electrical properties from $10^{-3}$ to $10^{+9} \mathrm{~Hz}-$ Physics and chemistry," in 2nd Int. Symp. On the Physics and Chemistry of Porous Media, Ridgefield, CT, Oct. 1986.

[22] G. R. Olheoft and D. E. Capron, "Laboratory measurements of the radiofrequency electrical and magnetic properties of soils from near Yuma, Arizona," U.S Geolog. Survey, Denver, CO, Open File Rep. 93-701, 1993.

[23] P. Parchomchuk, W. W. Wallender, and R. J. King, "Calibration of a waveguide sensor for measuring soil moisture," IEEE Trans. Geosci. Remote Sensing, vol. 28, pp. 873-878, Sept. 1990.

[24] N. R. Peplinski, F. T. Ulaby, and M. C. Dobson, "Dielectric properties of soils in the 0.3-1.3-GHz range," IEEE Trans. Geosci. Remote Sensing, vol. 33, pp. 803-807, May 1995.

[25] J. Sabburg, J. A. R. Ball, and N. H. Hancock, "Dielectric behavior of moist swelling clay soils at microwave frequencies," IEEE Trans. Geosci. Remote Sensing, vol. 35, pp. 784-787, May 1997.

[26] E. T. Selig and S. Mansukhani, "Relationship of soil moisture to the dielectric property," ASCE J. Geotech. Eng. Div., vol. GT8, pp. 755-770, Aug. 1975.

[27] S. S. Smith, "Soil characterization by radio frequency electrical dispersion," Ph.D dissertation, Univ. California, Davis, 1971.

[28] A. M. Thomas, "In situ measurement of moisture in soil and similar substances by 'fringe' capacitance," J. Sci. Instrum., vol. 43, pp. 21-27, 1966.

[29] G. C. Topp, J. L. Davis, and A. P. Annan, "Electromagnetic determination of soil water content: Measurements in coaxial transmission lines," Water Resources Res., vol. 16, pp. 574-582, June 1980.

[30] N. B. Troitskii and L. N. Stepanov, "Dependence of dielectric constant of soil on its specific surface," Soviet Agric. Sci., vol. 7, pp. 63-66, 1980.

[31] W. A. Wensink, "Dielectric properties of wet soils in the frequency range 1-3000 MHz," Geophys. Prospect., vol. 41, pp. 671-696, 1993.

John O. Curtis, photograph and biography not available at the time of publication. 